\title{
STATISTICAL SEARCH FOR MAGNETIC FIELD DECAY
}

\author{
F. Curtis Michel \\ Space Physics and Astronomy Department, Rice University
}

\begin{abstract}
The claims made in many statistical analyses for magnetic field decay follow from the assumption that radio luminosity declines slower than spin-down luminosity which is suggested by least-squares fits to the data. However, such fits are very sensitive to the behaviors of the fastest and slowest pulsars. If pulsar luminosities are plotted in distance-selected groups, the (radio inefficient) Crab and Vela pulsars are cleariy exceptional members and the remaining pulsars are consistent with a mean fixed conversion efficiency of $10^{-5}$. Numerical simulations (and theoretical analysis) strongly suggest that the pulsar period distribution peak is caused by luminosity selection and searches limited in distance by dispersion measure, and not by some mechanism that removes old pulsars. We cannot exclude magnetic field decay with some large time constant, $\geq 10^{7}$ years say, but only because it would make such a small difference to the expected pulsar statistics, not because it is required by the data.
\end{abstract}

\section{Introduction}

Magnetic field decay has been a popular issue, and repeated statistical analyses purport to determine a need for decay of pulsar magnetic fields on a time scale of several million years. It is an astonishing coincidence that such time scales are also typical of pulsar spin-down ages, which have to do with the magnetic field but not its decay rate. Thus if the putative decay rate had been much shorter, pulsars would fade at essentially constant spin rate and slowing dow'n would be an unimportant evolutionary factor, while if the decay rate had been much longer, pulsar evolution would be dominated entirely by spin down. Consequently, a magnetic field decay with the usual time constants quoted must statistically be in competition with spin down, and one wonders how, given two decay processes with similar time scales, one can so cleanly separate out magnetic field decay in the data.

The answer is simple: one assumes that the radio luminosity evolves differently from the spindown luminosity, $I \Omega \dot{\Omega}$ ! Specifically, it is assumed that the radio luminosity declines more slowly, in effect, than the spin-down luminosity. The consequence is that old pulsars are (by assumption) much brighter proportionately than they would be if the two luminosities simply tracked one another. The difference is critical because one can easily show that pulsars slowing down at constant conversion efficiency do not accumulate at long periods because they become too dim to be observed.

The assumption that the radio luminosity declines more slowly than the spin-down luminosity therefore creates a problem: accumulation of observable pulsars with long periods. The problem is then "solved" by turning these pulsars off (e.g., magnetic field decay).

Where then does the idea come from that "radio luminosity declines more slowly than spin-down luminosity?" Apparently it comes largely from a least-squares fit to the observationally biased data set of pulsar luminosities! The latter gives a "phenomenological" luminosity law $L_{\text {radio }} \approx L_{\text {total }}^{1 / 3}$ in rounded exponents (Lyne, Ritchings, and Smith 1975, Narayan and Vivekanand 1981, Prószyński and Przybycien 1984). If one looks at the data, this is a very weak correlation even in the available data set, dominated by the relative inefficiencies of the Crab and Vela pulsars.

In other words we are in effect saying, "The Crab and Vela pulsars are very inefficient, because they are rapid rotators, therefore the very slow pulsars must be very efficient." Of course we do not get to actually observe these supposedly "efficient" pulsars because their magnetic fields have supposedly decayed away. This is not the tightest logical argument. Moreover, we can go to the data and show that pulsars have, for all intents and purposes, roughly fixed efficiency at converting spin down luminosity into radio luminosity. Such a result should hardly be surprising, because the efficiency cannot exceed $100 \%$, so clearly there is going to be some physical limitation to how efficient the conversion can ever be (certainly not 100\%). That some individual pulsars might appear to be extremely inefficient is so unsurprising that one can hardly be bothered to trot out all the obvious possible factors that could explain such inefficiency. Beskin et al. (1984) have already pointed out that one gets a good representation of data on pulsars simply by adopting a fixed $10^{-5}$ efficiency. 


\section{Average efficiency}

We will now show that this efficiency is representative of the nearby pulsars neglecting the exceptional Crab and Vela pulsars, and is at least not implausible from a theoretical point of view [A pairproduction avalanche model gives just such values (Michel 1991).] Luminosity selection makes it difficult to deduce brightness distributions from the observed luminosities of pulsars, which also have a wide range of intrinsic luminosities. This bias can be illustrated by breaking up the pulsars according to distance, with an arbitrary definition of $<0.5 \mathrm{kpc}$ for "near" and using logarithmic intervals of 0.5 to $1.0,1.0$ to 2.0 , etc., thereafter. The results are shown in figure 1 [al! data discussed here are essentially from Manchester and Taylor (1981)]. First notice the radio luminosity, which is the agent

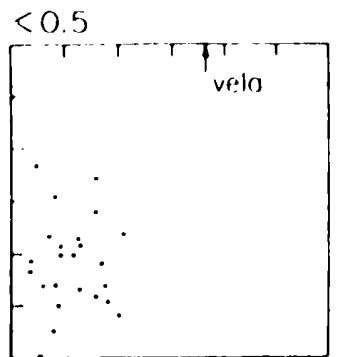

$0.5-1$

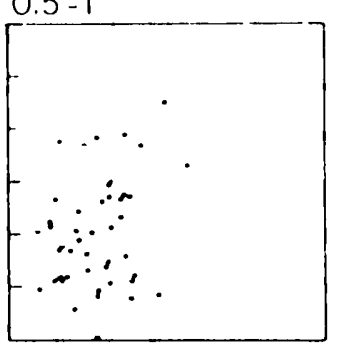

$1-2$

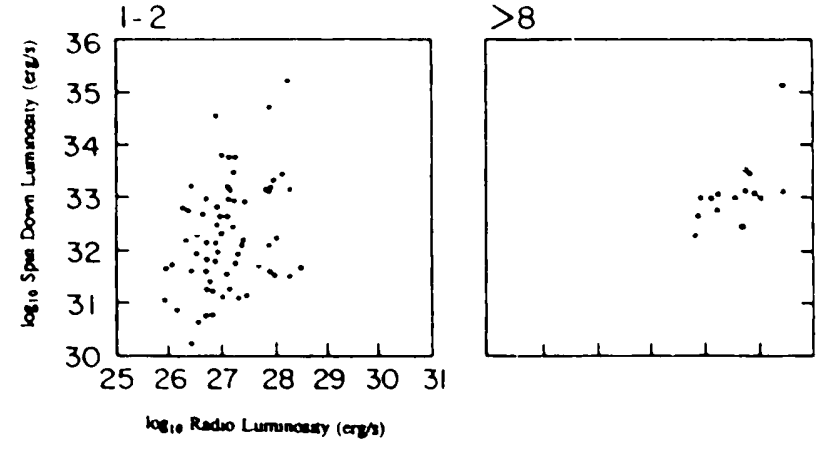

Figure 1 Spin-down luminosity vs. radio luminosity for logarithmic intervals of distance.

by which pulsars are usually detected at all. The nearby pulsars are seen to crowd against a limit of $10^{25} \mathrm{ergs} / \mathrm{s}$. In fact, no pulsar has a smaller luminosity in the Manchester and Taylor (1981) catalog. As we go to more distant pulsars, we can see an advancing vertical edge that cuts off everbrighter pulsars. Returning to the nearest pulsars, it is not entirely clear from this plot whether the luminosity "limit" at $10^{25} \mathrm{ergs} / \mathrm{s}$ is real or statistical. The distribution might appear more balanced perhaps with one or two pulsars to the left of the $<0.5 \mathrm{kpc}$ box, but that absence has little apparent statistical weight. Thus the failure to detect very weak pulsars seems a natural feature of the data, and one must wonder a little whether great efforts to identify a "turn-off" mechanism are really required. (There has been no shortage of proposed mechanisms, should such be required; several have even been suggested by this author.) We also see that each distance-grouped sample is cut off to the right as well. Just as for stars, the luminosity function is weighted heavily in favor of dim objects. In the case of pulsars, that weighting must be due in part to their slowing down with age. As we expand the volume, we steadily increase the chances of seeing the rare bright objects. Some of these may not be so distant, and hence their brightness may be overestimated. The brightest members of each group are selected with each step in distance. (The numbers are significantly more complete for the nearby pulsars than for the distant ones; the latter are dominated by Arecibo observations near $19^{h}$ right ascension and become increasingly incomplete with distance.)

Note that drawing a line in figure 1 running up at $45^{\circ}$ broadly typifies a radio luminosity versus total luminosity relationship fixed around $10^{-5}$. For the most distant pulsars, we begin to see a tendency for this line to bend over, which is once again suggestive of either the possibility of seeing such pulsars at much larger distances or a selection effect by which "distant" pulsars become more efficient by virtue of their not actually being that distant at all.

Figure 1 provides little rationale for insisting that radio luminosity evolves other than at roughly fixed efficiency. However, there is the adage that "I didn't see it until I believed it," and now that so may people believe, they manage to see evidence for magnetic field decay everywhere. Elsewhere (Michel 1990) I have tried to model numerically the various statistical arguments (galactic height distributions, luminosity functions, etc.), and I find that simple spin-down at fixed efficiency does such a good job that there is very little in the form of "residuals" left over to require anything else. Which is not to say that there are not other factors (beaming, drift out of galactic plane, etc.), but they do not improve upon the simulation of the actual data. Thus one cannot "invert" the data to say that they are required. 


\section{The period histogram}

Space does not permit reviewing the numerical simulations, so I will stick with one simple issue: Why is there a peak in the number of pulsar vs. period histogram (figure 2) at about $0.5 \mathrm{~s}$ ? Any power-

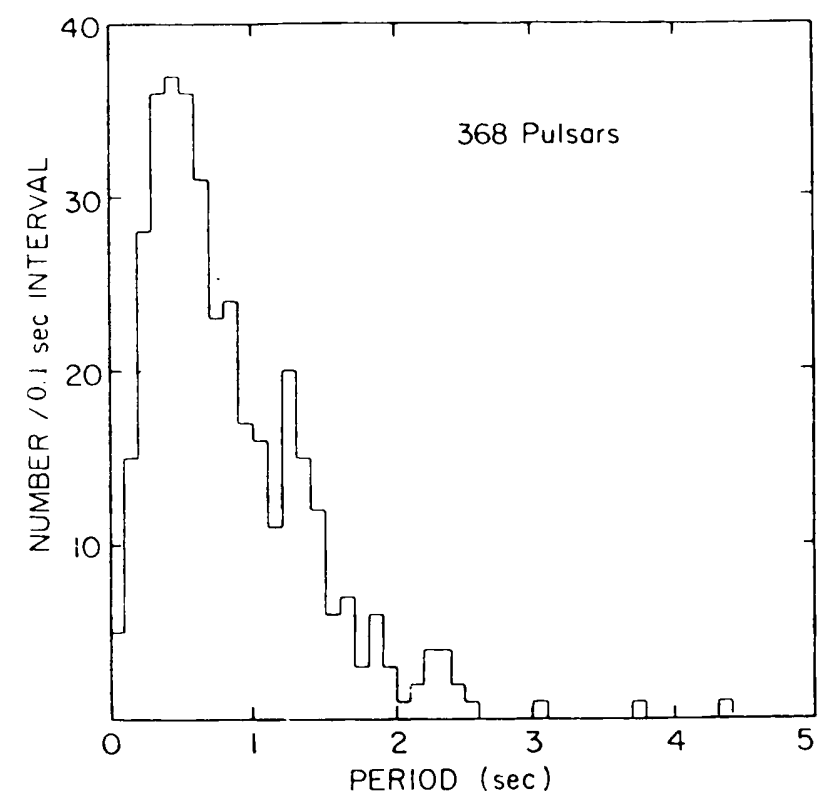

Figure 2 Observed histogram of the number of pulsars vs. period.

law evolutionary model would give $d N / d P \approx P^{\alpha}$ with the usual problem that we should either see hordes of fast ones $(\alpha<-1)$ or hordes of slow ones $(\alpha>-1)$. The answer (according to the simulations) is that there is a characteristic distance involved, more or less imposed by the increasing dispersion measure, beyond which detecting pulsars becomes extremely difficult. This is not a fixed number, of course, but for any given state of the art it will not be infinite but will be of some finite order of magnitude. Accordingly, for bright pulsars within this distance, we see every single one of them, and this gives the linear rise in numbers of pulsars with increasing period. On the other hand, this distance is irrelevant for the dim pulsars because we only see the nearby ones anyway, and the distribution falls off with a power law. The period at the peak in figure $2(0.5 \mathrm{~s})$ is that for which a typical $10^{12}$ Gauss pulsar would be just at the threshold detection flux density $\left(10^{25} \mathrm{erg} / \mathrm{s} \mathrm{kpc}^{2}\right)$ at the characteristic distance $(\approx 5 \mathrm{kpc})$. The simulations give figure 3. Thus the peak is, in a sense, an artifact, but an inevitable one because any presently plausible technology will always be distance and sensitivity limited. In fact, if we go fainter and deeper, the peak is likely to remain just about where it is!

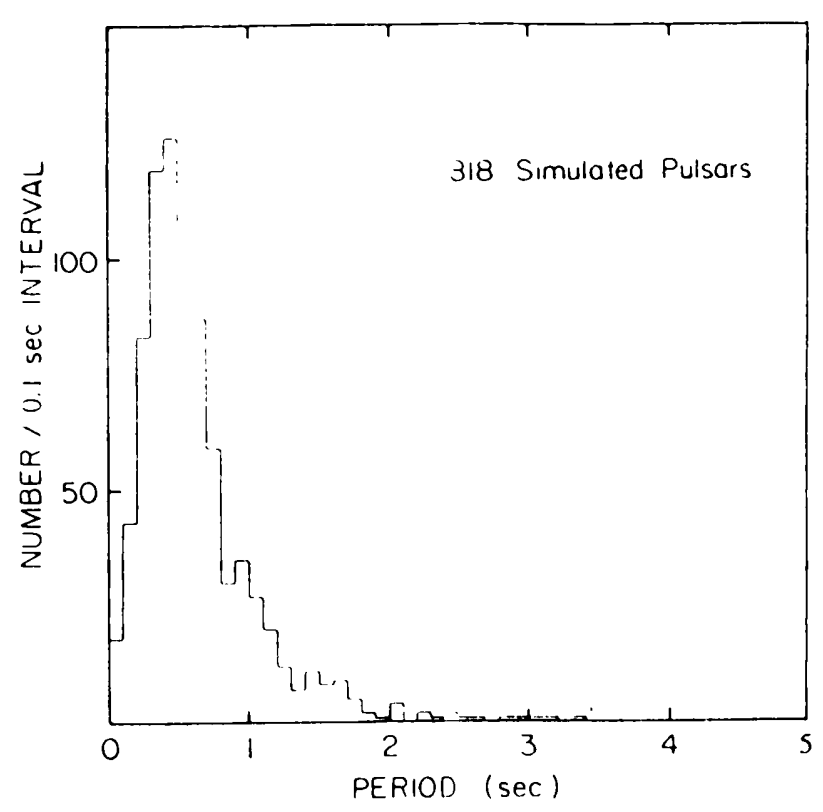

Figure 3 Simulated histogram of the number of pulsars vs. period.

\section{Theoretical analysis}

Let me close by explaining why the rise should be linear in figure 2 and is linear in figure 3 . If pulsars decay by pure magnetic dipole radiation without change in orientation, we have

$$
P(t)=P_{0}\left(\frac{t}{t_{0}}\right)^{1 / 2},
$$

where $t$ is the time since formation, with $t_{0}$ the present epoch and $P_{0}$ the typical period. The period derivative is then

$$
\dot{P}(t)=\frac{P_{0}}{2 t_{0}}\left(\frac{t}{t_{0}}\right)^{1 / 2}=\frac{P_{0}^{2}}{2 t_{0} P(t)} .
$$

Conservation of pulsar "flux" in time gives

$$
\frac{d N}{d P} \frac{d P}{d t}=\text { constant }
$$

where $N$ is the space density of pulsars, and because $\dot{P} \approx P^{-1}$, we can integrate to obtain

$$
N(<P) \approx P^{2}
$$

which can also be obtained by noting that the total number of pulsars is assumed to accumulate linearly with time, hence from eq.(1) as $P^{2}$. An important selection effect is luminosity, and the fixed efficiency assumption gives us $l_{\text {radio }} \approx P^{-4}$ while the received flux density falls as $S \approx L / d^{2}$. We can estimate the mean distance between pulsars as $d \approx N(<P)^{-1 / 2}$, so $S \approx P^{-3}$. What this means is that observable pulsars do not, as has been casually supposed, "accumulate" at long periods. [An important exception is the millisecond pulsars, which 
are not included in this pre-discovery data set: according to the simulations (Michel 1990), the finite age of the galaxy will be a minor factor in the number of observable pulsars with magnetic fields less than about $3 \times 10^{10}$ Gauss and a major factor for those with fields less than about $10^{9}$ largely because they spin down so slowly.]

We can rewrite eq.(4), using $L \approx P^{-4}$, as

$$
N(>L)=L^{-1 / 2} \text {. }
$$

The practical limit of flux that can be detected $\left(S_{0}\right)$ gives us a limit on the luminosity of pulsars that can be detected

$$
L<S_{0} r^{2} .
$$

The density of observable pulsars will then be, using the limit in eq.(6),

$$
N(<r) \approx \frac{1}{r}
$$

and the total will be

$$
n \approx \int N d V=\int N r d r \approx r .
$$

The number of observable pulsars is linearly proportional to how far we search, assuming a disk population and a given limiting $S_{0}$. For short periods, we obtain $d n / d P \approx P$, consistent with differentiating equation 4 and having no distance selection. The long-period pulsars are entirely luminosity selected, giving

$$
\frac{d n}{d p} \approx \int_{0}^{r} \frac{d N}{d P} r d r
$$

with $d N / d P \approx P$ as before, but because $L \approx P^{-4}$ and the limiting distance is defined by $L \approx S_{0} r^{2}$, we have $r \approx P^{-2}$, which then gives

$$
\frac{d n}{d p} \approx \frac{1}{P^{3}}
$$

In other words, the number observable per unit period interval climbs linearly and then falls off as the inverse cube.

\section{Conclusions}

Magnetic field decay is a perfectly plausible physical phenomenon, and we would be remiss if we left the impression that we have interpreted the data to say that such decay does not take place in pulsars. The point is that the spin-down evolution at constant efficiency gives such a good fit that there is little left to be pulled out of the data. Other effects can be added (e.g., drift out of the galactic plane) without much overt effect, so one cannot "prove" that pulsars drift out of the galactic plane, although some must given their high space velocities. We know of this effect not from the statistical data but from independent observational information. In the same way, it is correspondingly hard to "exclude" magnetic field decay, especially at some longish time constant. We do, however, have independent information. If, for example, the cyclotron lines in gamma-ray bursters are correctly interpreted as electrons in teragauss magnetic fields, and if these objects are indeed old pulsars (Michel 1985), any magnetic decay time constant must be comparable to a Hubble time. Only if one introduces assumptions that keep spin-down from depopulating the observable pulsar population (e.g., little or no decline in radio luminosity) does one require a separate mechanism to turn off the old "bright" pulsars. 